\section{Wo bleibt das Verursacherprinzip?}

\author{
Das Erneverbare-Energien-Gesetz und der Handel mit Ökostrom sind Maß- \\ nahmen, um den Anteil regenerativer Energien am Stromverbrauch zu erhöhen. \\ Ob der Kauf von im Vergleich zu herkömmlichem Strom teurere Ökostrom aber \\ einen sinnvollen Beitrag zum Klimaschutz leistet, ist umstritten.
}

A Von Florian Wetzig nfang April passierte das Erneuerbare-Energien-Gesetz (EEG) den Bundestag und nimmt momentan die letzten parlamentarischen Hürden. Beim EEG werden die Mehrkosten, die die regenerative Stromerzeugung gegenüber der konventionellen verursacht, von allen Stromverbrauchern gemeinsam getragen.

Gleichzeitig entschließen sich Verbraucher, ihrem ursprünglichen Stromversorger den Rücken zu kehren und Ökostrom, Strom aus regenerativen Energien zu kaufen. Sie sind bereit, persönlich einen höheren Preis für solchen Strom zu zahlen, der für sie in regenerativ betriebenen Kraftwerken erzeugt wird. Das dies zum Klimaschutz beiträgt, suggerieren die Ökostromhändler ihren Kunden. Ob dies wirklich der Fall ist, fragen sich die Verbraucher nur selten, aber zu Recht (1).

Beide Instrumente, EEG und Ökostromhandel, haben eine Erhöhung der regenerativen Stromerzeugung und dementsprechend eine Verringerung der Kohlendioxid-Emissionen zum Ziel, nur sind die Wege unterschiedlich. Während das EEG ein gesellschaftliches, politisches Instrument zur Förderung der Erneuerbaren Energien darstellt, ist der Ökostromhandel von der Motivation und Zahlungsbereitschaft der einzelnen Stromkunden abhängig.

\section{Funktionsweise}

Die Funktionsweise des EEG ist einfach: Betreiber von Anlagen zur regenerativen Stromerzeugung speisen Strom aus Erneuerbaren Energien in das Stromnetz ein und erhalten vom Netzbetreiber eine gesetzlich festgelegte Vergütung. Der eingespeiste Regenerativstrom wird auf alle Stromverbraucher in Deutschland verteilt. Die Summe der Vergütungszahlungen wird zunächst durch eine Ausgleichsregelung anteilig von allen Elektrizitätsversorgungsunternehmen (EVU) und über die Stromrechnung schließlich von deren Kunden also letztlich ebenfalls allen Stromverbrauchern - getragen.
Anders beim Ökostromhandel: Ein Ökostromkunde schließt einen Versorgungsvertrag mit einem Ökostromhändler ab, der die nachgefragte Menge an regenerativ erzeugtem Strom in das Stromnetz einspeist und zum Verbraucher „,durchleitet“. Hier trägt allein der Kunde die im Vergleich zur konventionellen Stromerzeugung meist höheren Kosten der regenerativen Stromerzeugung. Die Motivation zum Kauf von Ökostrom ist dabei in der Regel der Wunsch, zur Verringerung der Kohlendioxid-Emissionen beizutragen. Ob der Kauf eines Ökostromproduktes tatsächlich eine Verringerung der Kohlendioxid-Emissionen bewirkt, kann keinesfalls a priori als gesichert angesehen werden.

Nur ein Ökostromprodukt, das zu einer Mehrproduktion an regenerativ erzeugtem Strom führt, bewirkt eine Verringerung der fossilen Stromerzeugung und führt dadurch zu einer Reduzierung der Kohlendioxid-Emissionen. Eine Mehrproduktion an Ökostrom setzt daher grundsätzlich voraus, dass der bezogene Ökostrom aus Neuanlagen stammt, Anlagen, die ohne die Zahlungsbereitschaft der Ökostromkunden nicht errichtet worden wären. Der Kauf von Ökostrom, der in bereits bestehenden Anlagen erzeugt wird, ist demzufolge nicht mit einem positiven Umweltnutzen verbunden. Unterschiedliche Institutionen beschäftigen sich derzeit mit dieser Problematik. Sie vergeben Label an solche Ökostromprodukte, die bestimmte Maßgaben

Inserieren Sie
in Ökologisches Wirtschaften!
Sprechen Sie genau Ihre Zielgruppe an.
Erscheint 5 x jährlich.
Fordern Sie noch heute die Mediadaten an!
Frau Dagmar Huber berät Sie gerne.
Kontakt:
Dagmar Huber
Waltherstraße 29, 80337 München
Fon 089/544 184 12, Fax 089/544 184 49
E-Mail huber@oekom.de

erfiillen, wobei gerade der Anteil an Strom aus Neuanlagen eines Ökostromproduktes vielfach ein zentrales Bewertungskriterium zur Bemessung des Umweltnutzens eines Produktes darstellt.

Die Mehrkosten der EEG vergüteten regenerativen Stromerzeugung werden anteilig von allen Stromverbrauchern in Deutschland getragen. Die durch das EEG initiierte Verringerung der KohlendioxidEmissionen wird damit von denjenigen finanziert, die für die Schäden der konventionellen Stromerzeugung verantwortlich sind, womit das EEG als mit dem Verursacherprinzip vereinbar bezeichnet werden kann.

Entscheidet sich ein Kunde für den Kauf von Ökostrom und bewirkt dadurch annahmegemäß eine Verringerung der Kohlendioxid-Emissionen, so wird er für sein „,klimafreundliches“ Verhalten nicht belohnt, sondern muss in der Regel einen höheren Preis bezahlen, als die Verbraucher, die konventionell erzeugten Strom beziehen. Es kommt also zu einer Umkehrung des Verursacherprinzips: Nicht diejenigen, die für die Schäden der konventionellen Stromerzeugung, wie Klimawandel und die Probleme der Entsorgung von radioaktivem Material verantwortlich sind, kommen für die entstehenden Kosten auf. Statt dessen werden Verbraucher, die durch ihre Kaufentscheidung eben diese Schwierigkeiten zu vermeiden helfen, belastet. Letztere setzen sich aber für die Vermeidung von Umweltschäden ein, die letztlich allen - auch den Käufern von konventionell erzeugtem Strom - zu Gute kommen.

\section{"Und" oder "oder"}

Während die isolierte Betrachtung von EEG und Ökostromhandel bestätigt, dass sowohl das EEG als auch der Ökostromhandel potentiell zu einer Erhöhung der regenerativen Stromproduktion führen können, wobei dies wie oben dargestellt hinsichtlich des Ökostromhandels nicht zwangsläufig der Fall ist, stellt sich nun die Frage nach dem Zusammenspiel der beiden Instrumente. Können sich EEG und Ökostromhandel als Instrumente zum Klimaschutz sinnvoll ergänzen? Auf den Punkt gebracht: Kann der Ökostromhandel neben der Förderung regenerativer Stromerzeugung durch das EEG zusätzliche Erzeugungspotentiale wecken und dadurch einen zusätzlichen Beitrag zum Klimaschutz leisten? Entscheidendes Kriterium zur Beantwortung dieser Frage sind die Spezifika derjenigen Anlagen, in denen der als Ökostromprodukt verkaufte Regenerativstrom erzeugt wird und 
die Kosten der Stromerzeugung in den jeweiligen Anlagen. Es gilt an dieser Stelle zwischen zwei Anlagenkategorien zu unterscheiden.

Der ersten Kategorie entsprechen solche Ablagen, die den EEG-Kriterien (hinsichtlich Energieträger, Größe etc.) entsprechen und Erzeugungskosten je Kilowattstunde verursachen, die den EEG-Vergütungssätzen entsprechen oder diesen unterschreiten. Der Kauf eines Ökostromproduktes aus derartigen Anlagen führt nicht zu einer zusätzlichen Erzeugung von Regenerativstrom. Die Erzeugungsanlagen könnten auch ohne die Nachfrage des Ökostromkunden kostendeckend betrieben und der Strom nach EEG eingespeist werden. Zu beachten ist darüber hinaus die Finanzierungsseite: Wäre der Strom statt des Verkaufs als Ökostromprodukt nach EEG eingespeist worden, hätten aufgrund der EEG-Ausgleichsregelung alle Stromverbraucher die Finanzierungslast getragen. Durch den direkten Verkauf des Stroms werden die Erzeugungskosten aber nicht von allen Stromverbrauchern, sondern von den Ökostromkunden getragen. Die Allgemeinheit wird beim Kauf eines Ökostromproduktes aus der entsprechenden Anlagenkategorie also sogar entlastet. Die zweite Anlagenkategorie stellen Anlagen dar, die den Kriterien nach EEG nicht entsprechen und/oder Erzeugungskosten je Kilowattstunde verursachen, die die EEG-Vergütungssätze überschreiten. Der Kauf eines Ökostromproduktes aus solchen Anlagen führt zu einer zusätzlichen Erzeugung von Regenerativstrom über die durch das EEG hervorgerufene Ökostromproduktion hinaus. In beiden Fällen ist jedoch fraglich, ob eine Förderung entsprechender Anlagen sinnvoll ist: Die Förderung sehr unwirtschaftlicher Anlagen ist grundsätzlich als ökonomisch ineffizient zu bezeichnen. Solche Anlagen, die die Kriterien nach EEG nicht erfüllen, stehen im Verdacht, ökologisch bedenklich zu sein.

\section{Fazit}

Der Ökostromhandel ist somit als Ergänzung zum EEG nicht geeignet, zu einem nennenswerten zusätzlichen Ausbau der regenerativen Stromerzeugung beizutragen. Im schlechtesten Fall führt der Kauf von Ökostrom zu keinerlei zusätzlicher Ökostromproduktion und hat damit keine Reduzierung der Kohlendioxid-Emissionen zur Folge. Findet eine zusätzliche Ökostromproduktuion statt, bedeutet dies nur in wenigen Fällen eine Produktion über die durch das EEG hervorgerufene Erzeugung hinaus, wobei dies dann durch hohe Kosten und/ oder möglicherweise bedenkliche ökologische „Nebenwirkungen“ erkauft werden muss. In jedem
Fall aber tragen die Käufer von Ökostrom einen höheren Preis für ein Produkt, dessen Vorteile letztendlich allen zur Verfügung stehen. Und - was viel gravierender ist - es werden die Käufer von konventionellem Strom an den Kosten für den Klimaschutz nicht beteiligt.

Es stellt sich die Frage nach einem Ausweg. Wünschenswert ist eine Möglichkeit für den Verbraucher, eine tatsächliche Mehrproduktion an Ökostrom zu erreichen und dadurch seinen Beitrag zum Klimaschutz leisten zu können, ohne gleichzeitig das Verursacherprinzip aushebeln zu müssen und letztlich ,als der Dumme dazustehen“. Diese Möglichkeit ist vorhanden:

Durch eine Investition in Anlagen zur regenerativen Stromerzeugung kann jeder Stromverbraucher den Ausbau der regenerativen Stromerzeugung vorantreiben, bei zweifachem Vorteil gegenüber dem Ökostromhandel: Es kommt in jedem Fall zu einer Mehrproduktion an regenerativem Strom und die (Mehr-)Kosten der regenerativen Stromerzeugung zahlen alle. Durch die finanzielle Beteiligung an einer Bürgersolaranlage, einem Bürgerwindpark oder ähnlichen Vorhaben, die in der Regel schon mit sehr geringen Investitionssummen möglich ist, ist dabei sogar noch eine Verzinsung des eingesetzten Kapitals möglich, die an anderer Stelle für eine nachhaltige Wirtschaftsweise eingesetzt werden kann. Der positive Werbeeffekt solcher Modelle ist die steigende Akzeptanz erneuerbarer Energien durch Partizipation. Der erzeugte Strom wird nach EEG eingespeist und die Kosten der regenerativen Stromerzeugung somit von allen Stromverbrauchern getragen.

Dies stellt vor dem Hintergrund der derzeitigen Förderung regenerativer Stromerzeugung durch das EEG eine sowohl ökologisch sinnvolle als auch ökonomisch gerechte Möglichkeit für den einzelnen dar, sich über die staatlichen Aktivitäten hinaus individuell für eine umweltfreundliche Stromerzeugung einzusetzen.

\section{Anmerkung}

(1) Wetzig, F.: Ökostromhandel und das EEG. Dipl.Arbeit, Kiel 2001.

\section{Der Autor}

Florian Wetzig ist wissenschaftlicher Mitarbeiter im Bereich Klimaschutz und nachhaltige Energiewirtschaft am Institut für ökologische Wirtschaftsforschung (IÖW).

Kontakt: IÖW, Potsdamer Str. 105, 10785 Berlin. Tel. 030-884594-40, E-Mail: florian.wetzig@ioew.de

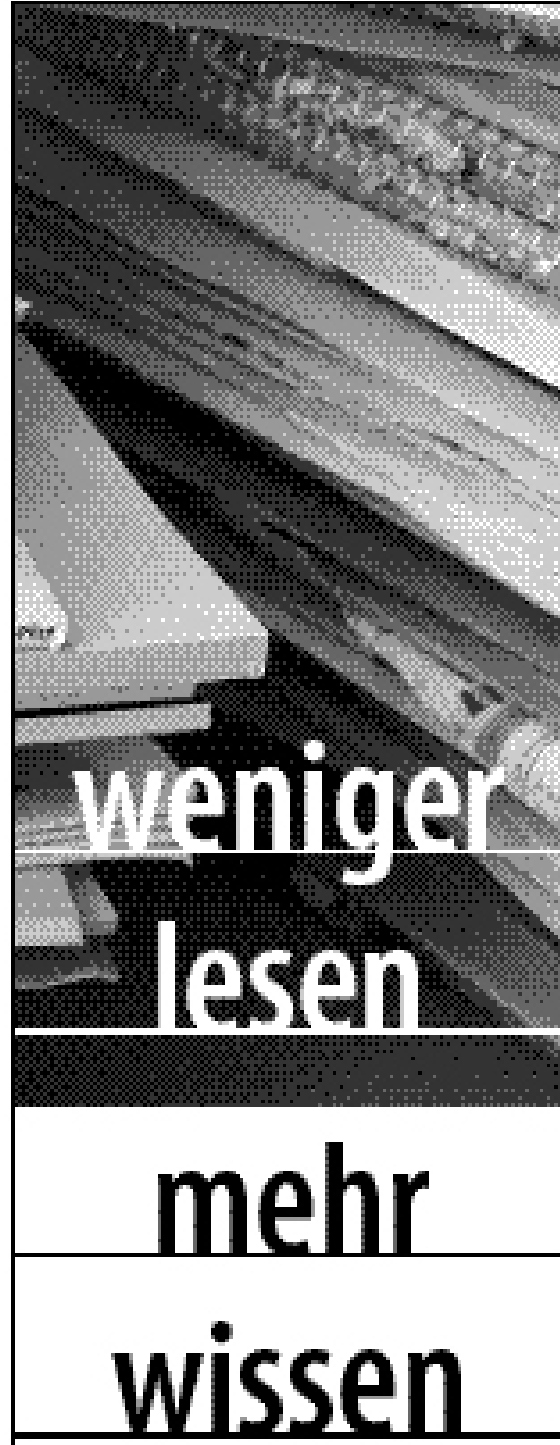

Barichte, Hintergrtinde, News zn

Ölologie und Nachhaltigkeit.

Von der lodalen Bbone bis zur

inkenationalen Politik.

Lesen Eie jeden Mornt, was wichtig ist.

Und wa wichtig wird.

Uber 5.000 Aktive und Entscheider in

Deutschland, Osterreich

und der Sctmeiz lesenpunkt.um.

Und wissen mehr.

\section{Probeheft?}

www.oekcom.de'punktum

\section{punkt.um}


(c) 20I0 Authors; licensee IÖW and oekom verlag. This is an article distributed under the terms of the Creative Commons Attribution Non-Commercial No Derivates License (http://creativecommons.org/licenses/by-nc-nd/3.o/), which permits unrestricted use, distribution, and reproduction in any medium, provided the original work is properly cited. 